tion in a whole plant (Professor A. J. Vlitos, Tate and Lyle, Ltd, Keston), and some aspects of growth control in cereal seedlings (Dr L. L. Mer, Imperial College, London).

The transport of auxins, gibberellins and cytokinins was discussed by several participants. Professor W. P. Jacobs (Princeton University) presented evidence for the polar transport (in Coleus) of all of the three classes of growth regulators. Professor T. K. Scott (University of North Carolina) demonstrated that the movement of gibberellins in sugarcane tissues is strongly influenced by auxins and perhaps also by other endogenous growth regulators. $\mathrm{Dr} \mathrm{H}$. Kaldewey (University of Saarlandes) presented good evidence for polar movement of radioactive ${ }^{14}$ IAA in the stems and in the roots of intact cotton seedlings. An original concept for explaining apical dominance was presented by Professor Y. Vardar (Ege University, Turkey). Professor H. Bürstrom (University of Lund) demonstrated quite conclusively that the anatomy (that is, cell type) of a given tissue determines to a considerable extent the velocity of movement of growth regulators through the tissue. $\mathrm{He}$ also demonstrated the histological effects on cells after treatment with various classes of growth regulators.

Interactions between the various classes of endogenous growth regulators were reviewed by Professor A. W. Galston (Yale University) and by Professor A. C. Leopold (Purdue University). Both speakers stressed that auxins, gibberellins, cytokinins, abscisic acid and ethylene operate in a dynamic system where the presence of one type of growth regulator influences the physiological activity of another. Auxin, for example, may predispose a cell to respond to a gibberellin, and in some instances treating a tissue with a gibberellin induces it to produce more auxin; in turn this may lead to a release of ethylene. Ethylene may produce a host of physiological responses including the induction of flowering in tissue cultures of higher plants as was demonstrated by Dr C. Nitsch (CNRS, Gifsur-Yvette).

Dr O. M. Heide (Makerere University) reviewed some of the physiological effects of cytokinins with special reference to the induction of rooting and initiation of buds. He demonstrated that these effects were mediated by temperature. Of particular interest was the demonstration that rooting of cuttings can be stimulated by cytokinins under certain conditions.

The role of ethylene in plant growth was discussed in some detail by $\operatorname{Dr} \mathrm{S}$. P. Burg (University of Miami). As one delegate remarked, "Ethylene can do anything an auxin can do and even better". Dr Burg demonstrated that ethylene is indeed a very active plant growth regulator but an extremely difficult and elusive compound to study, chiefly because it is a gas.

A thorough review of the auxins and other auxin-like substances by Professor K. V. Thimann (University of California, Santa Cruz), followed by a more specific contribution by $\mathrm{Dr}$ J. ShenMiller (Argonne National Laboratory), set the stage for lively discussions on whether the classical auxins, as studied in isolated plant parts and in dark rooms, are as relevant to the growth of intact plants as was once thought. Dr Mer indicated, in discussion, that some of the published results may be interpreted in more than one way and that extrapolations to whole plant physiology from such results are invalid.

Inhibitors of growth, some new synthetic growth regulators and some interesting and aberrant physiological effects of auxins on growth were also discussed. Dr K. Dörffling (State Institute for General Botany and Botanic Garden, Hamburg) reviewed the status of abscisic acid as a naturally-occurring plant growth regulator.

A thorough description of the synthetic growth regulators, known as morphactins, was presented by $\operatorname{Dr} G$. Schneider (E. Merck AG, Darmstadt). These substances retard the growth and development of dicotyledonous species and in the future may have important practical applications.

Professor L. Lona (University of Parma) presented evidence that indole3-butyric acid (IBA) acts as a gibberellin-like substance in certain species. $\mathrm{He}$ stressed the need for evaluating the physiological effects of any given growth regulator on a wide range of species before attempting to classify the regulator.

\title{
Heterogeneous Nucleation from a Polymer Melf
}

THE ingenious researches of David Turnbull in the nineteen-fifties stimulated a great deal of work on the mechanisms and temperature dependence of nucleation in supercooled metallic and molecular melts. The classical Becker-Volmer theory of homogeneous nucleation is now firmly established for these categories of materials, but at the same time it has become clear that nuclei form homogeneously only under very exceptional circumstances: normal melts contain specks of impurity that act as nucleation catalysts. A fortiori, crystals forming from polymer melts would be expected to be heterogeneously nucleated always, in view of the extreme improbability that a sufficient length of polymer chain should align spontaneously in the correct configuration to constrain further growth in the proper pattern. But so far no heterogeneous nucleation catalyst for a polymer has been properly characterized and, generally speaking, this aspect of polymer science is confused.

In next Monday's Nature Physical Science, S. Y. Hobbs casts some light on this problem. Hobbs crystallized spherulites of isotactic polypropylene from the melt, using short lengths of carbon fibre as nucleation catalysts. These were standard 'Morganite' fibres made from a polyacrylonitrile precursor, and two fibre variants were used -high strength (type 1) and high modulus (type 2). Type 1 fibre is known, from electron microscopy and small-angle X-ray scattering, to consist of very small turbostratic graphite crystallites of random orientation, whereas type 2 contains larger, highly oriented crystallites, with the basal graphite planes parallel to the fibre axis. Hobbs found that type 2 fibres were highly efficient nucleation catalysts, whereas type 1 fibres were ineffective.

The polymer chains in the crystalline structure of polypropylene are arranged in straight spirals. Hobbs showed that such a spiral can be accurately keyed to a graphite basal plane through some of the hydrogen atoms which (according to recent molecular orbital calculations) are preferentially chemisorbed at the midpoints of $\mathrm{C}-\mathrm{C}$ bonds in the graphite structure. For effective nucleation the spiral has to be "set", perhaps along one complete repeat period, and this requires the graphite basal plane which serves as substrate to exceed a certain minimum dimension, which Hobbs estimates at $50 \AA$. In type 2 carbon fibres, the constituent crystallites are more than $100 \AA$ across measured in the basal plane, whereas in type 1 they are only $\sim 25 \AA$ across. The inefficacy of type 2 fibres is thus readily understood.

Type 2 carbon fibres have a crystallographic "fibre texture", which implies that the normals to the basal plane are randomly distributed in a plane normal to the fibre axis. It would be interesting to continue Hobbs's work with single crystals of graphite or alternatively with highly oriented pyrolytic graphite. On the basis of Hobbs's interpretation of his observations, polypropylene would be expected to crystallize on such a substrate in twodimensional spherulites, all polymer spirals being constrained to lie parallel to the exposed graphite basal plane. This form of nucleation would generate a unique form of preferred orientation with, presumably, strongly anisotropic properties. 